\title{
CARBON STARS IN THE SMC
}

\author{
D.H. MORGAN ${ }^{1}$ and D. HATZIDIMITRIOU ${ }^{2}$ \\ ${ }^{1}$ Royal Observatory, Blackford Hill, Edinburgh EH9 3HJ, U.K. \\ ${ }^{2}$ University of Crete and Institute of Electronic Structure and Laser, P.O. Box 1527, \\ GR 711-10 Heraklion, Crete, Greece
}

\begin{abstract}
More than 1000 new carbon stars have been identified in a $\sim 200$ deg $^{2}$ area of the SMC during a survey of UK Schmidt Telescope objective-prism plates. Their spatial distribution is like that of the red horizontal branch stars.
\end{abstract}

\section{Introduction}

The carbon stars are considered as belonging to a rather broadly defined, intermediate age population and are often used as tracers of such populations in nearby galaxies. In the Magellanic Clouds in particular, the plethora of carbon stars (coupled with their intrinsic brightness) renders them particularly useful test particles for kinematical and dynamical studies of their parent galaxies. In an attempt to reveal the distribution of the intermediate-age population over the entire SMC, we have undertaken a survey of carbon stars covering $\sim 200 \mathrm{deg}^{2}$, improving by fortyfold on the spatial coverage of previous catalogues. (See Azzopardi [1993] for a review of older catalogues.) Preliminary results of this study are presented here.

\section{The Dataset}

The carbon stars were identified by visually scanning seven deep objective-prism plates which were taken with the $1.2 \mathrm{~m} \mathrm{UK} \mathrm{Schmidt} \mathrm{Telescope} \mathrm{and} \mathrm{cover} \mathrm{a} \mathrm{total} \mathrm{area} \mathrm{of} 15^{\circ} \times 15^{\circ}$ on the sky. The innermost area $\left(2^{\circ} \times 2^{\circ}\right)$ surveyed by Rebeirot et al. (1993 - RAW93) was mostly excluded from the search because of the forbidding number of overlapping of images. The carbon star spectra were selected on the basis of their pronounced Swan $C_{2}$ bands at $4737 \AA$ and $5165 \AA$. The appearance of the spectra is very characteristic and the great majority of the candidates should be bona fide carbon stars. The full catalogue (Morgan \& Hatzidimitriou, in preparation) will be published elsewhere and will include a detailed comparison with RAW93. 


\section{Results}

More than 1000 new carbon star candidates are included in the new catalogue. Figure 1 shows the surface distribution of the carbon stars detected in both the present survey and RAW93. In this diagram, the SMC appears elliptical, aligned with the Bar, but with significant distortion of the outer contours in the northward direction in particular, so much so that the overall shape of the outer contours no longer resembles that of the inner contours. This result matches the distribution of old populations in the SMC revealed in Gardiner \& Hatzidimitriou (1992 GH92). The diameter of the outermost contour is of the order of $11.5^{\circ}$; it is the first time that the spatial extent of the stellar component of the SMC has been revealed out to such distances. Also, as in the GH92 data, the low surface density outer region is much less extensive in the W and SW. Another interesting feature of Fig. 1 is the southward, arm-like extension of the carbon star distribution at (projected) distances larger than $4^{\circ}$ (approximately $4 \mathrm{kpc}$ ) from the optical centre. The final point of note is that there are isolated carbon stars found to the $E$ of the SMC, towards the LMC. Others are found farther E still, beyond the boundary of Fig. 1.

The carbon star distribution follows quite closely the distribution of clump/HB stars as would be expected if the majority of the carbon stars are older than $\simeq 1-2$ Gyr and is quite different from the distribution of younger stars (<1Gyr) - see GH92. Figure 2 compares the radial distribution of clump/HB stars from the SMC centre northwards with that of the carbon stars in the same direction. The similarity of the two distributions is apparent. The carbon star distribution is more extended in the NS direction, but this is most probably due to the more limited spatial coverage of the GH92 study. For the same reason, the southward arm-like feature of the carbon star distribution is not shown by the clump/HB stars.

The resemblance between the distributions of the two population groups indicates that either the intermediate age ( $<8 \mathrm{Gyr}$ as typified by the carbon stars) and older populations $(<12 \mathrm{Gyr}$ clump/HB stars) are well mixed spatially, or the former age group is predominant over older stars of ages 8-12 Gyr.

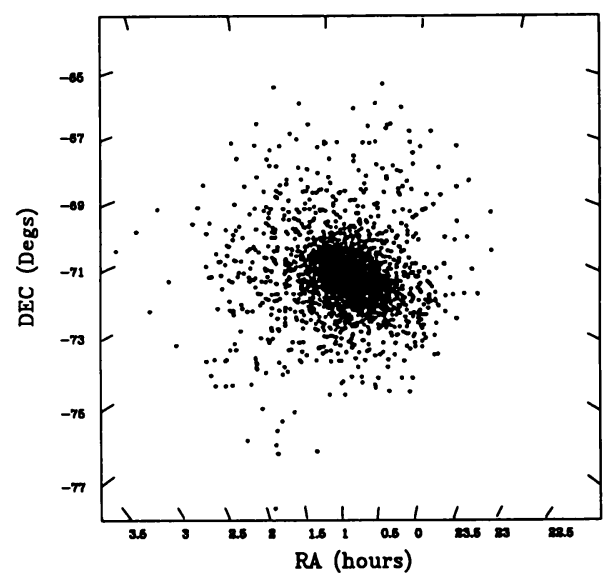

Figure 1. The carbon stars in the SMC. The points shown are taken from the present work and from RAW93. 


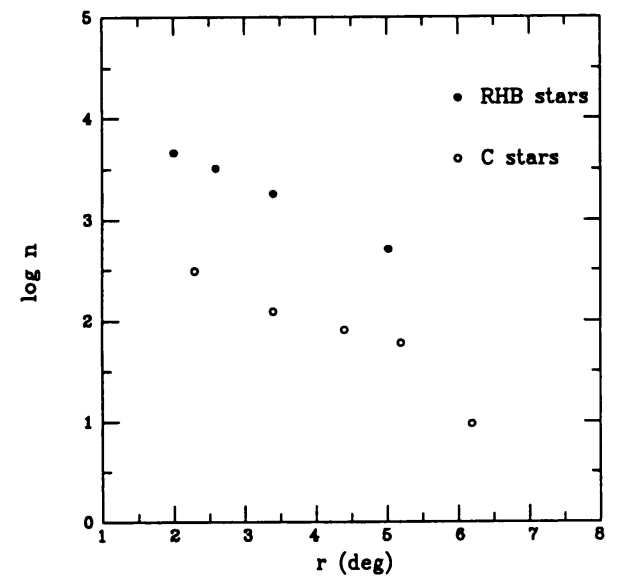

Figure 2. The surface densities of carbon stars and RHB stars (from GH92) as functions of distance from the SMC centre. (The ordinate zero points are not absolute).

\section{References}

Azzopardi, M., 1993. In 'New aspects of Magellanic Cloud Research', eds. B. Baschek, G. Klare and J. Lequeux, Proc. Heidelberg, Germany, p. 86.

Gardiner, L.T. and Hatzidimitriou, D., 1992. Mon. Not. R. astron. Soc., 257, 195. (GH92).

Rebeirot, E., Azzopardi, M. and Westerlund, B.E., 1993. Astron. Astrophys. Suppl., 97, 603. (RAW93). 